\title{
Editorial: Mental practice: clinical and experimental research in imagery and action observation
}

\author{
Magdalena letswaart ${ }^{1 *}$, Andrew J. Butler ${ }^{2}$, Philip L. Jackson ${ }^{3}$ and Martin G. Edwards ${ }^{4}$ \\ ${ }^{1}$ Psychology, University of Stirling, Stirling, UK, ${ }^{2}$ Neuroscience Institute, Georgia State University, Atlanta, USA, ${ }^{3}$ École de \\ Psychologie and CIRRIS and CRIUSMQ, Université Laval, Québec, Canada, ${ }^{4}$ Institut de Recherche en Sciences \\ Psychologiques, Université Catholique de Louvain, Louvain-la-Neuve, Belgium
}

Keywords: mental practice, action simulation, action observation, imagery, cognitive neuroscience

\section{OPEN ACCESS}

Edited and reviewed by: Hauke R. Heekeren, Freie Universität Berlin, Germany

*Correspondence:

Magdalena letswaart magdalena.ietswaart@stir.ac.uk

Received: 22 June 2015 Accepted: 01 October 2015 Published: 15 October 2015

Citation:

letswaart M, Butler AJ, Jackson PL and Edwards MG (2015) Editorial:

Mental practice: clinical and experimental research in imagery and action observation

Front. Hum. Neurosci. 9:573. doi: 10.3389/fnhum.2015.00573
This editorial accompanies 18 articles as part of a Frontiers research topic. The aim of this research topic was to clarify the underlying mechanisms involved in mental practice of action, bringing together evidence from a range of disciplines including cognitive neuroscience, experimental neuropsychology, sport and movement science, clinical neuropsychology and clinical neurology. The need to clarify the underlying mechanisms of mental practice is a pressing one. Mental practice of action has been explored in sport psychology for several decades, with the aim to use mental practice to improve sport performance. However, following the discovery of the mirror neuron system (see for example, Rizzolatti and Craighero, 2004), the perspective of mental practice has changed to a rationale based on neuroscience and to research focussed on understanding the neural processes of mental practice. Evidence that the brain simulates action has resulted in a common understanding of "functional equivalence" (Jeannerod, 1994): the idea that the mental representation of an action or percept in the person's mind is the neural "equivalent" to the physical action or actual percept. This ability to mentally represent action using the motor system allows for action simulation, providing conscious mental rehearsal of movement (imagery), but also allows for a common percept when observing the movements of others. Finally, in recent years, the disciplines of clinical neuropsychology and neurology have begun to use mental rehearsal of action, or mental practice, to produce improvements normally attributed to practicing actual movements.

At the heart of all of the research is the idea that mental practice of action uses equivalent neural processes to those used in action execution. Of course, there is debate on what one understands to be "equivalent," but the common reasoning seems to be that because mental practice (motor imagery and action observation) is functionally or neurally equivalent to actual practice, the efficacy principle of mental practice is that the motor areas are "trained," perhaps through Hebbian learning "firing-rewiring." Although the scientific foundation of this idea of action simulation is very sound in neuroimaging research (e.g., Sharma and Baron, 2013, this issue), the link to behavioral evidence or efficacy is currently weak. The neural correlates of mental practice are just that: correlates and do not justify inference about function, efficacy, or critical causality. There nevertheless seems to be reluctance in the field to address the underlying mechanisms of mental practice efficacy. This comes maybe as no surprise. A functional equivalence rationale for mental practice is intuitive and appealing and will therefore attract interest and funding. It is hardly in the researchers' interest to potentially undermine the idea by getting to the bottom of the matter.

We are now 15 or maybe 20 years into mental practice efficacy research based on the neural equivalence premise (Jackson et al., 2001). What is apparent is that the above simple interpretation of equivalence is not reflected in emerging data. It seems that mental practice efficacy is much more complex than simple Hebbian learning. There may be an analogy with the development of our understanding of the supplementary motor area (SMA) over that same time period. Initially SMA was thought of as a simple planning neural strip, but we have since understood the operation 
of SMA to be highly complex in the way it is involved in inhibition. For example, in studies using fMRI, motor imagery and action observation often do not activate the primary motor cortex (M1) because the SMA is thought to supresses the M1 activity (presumably preventing the individual from actually executing actions). The inherent role of inhibition in mental practice and the complexity of efficacy mechanisms still require further research. The popular notion that anything to do with the mirror neuron system is a simple matter of equivalence, or similarly that in applied contexts of mental practice equivalence is the end of the conversation, needs to change. We now need to go beyond what we are comfortable with and challenge what we know, even if we risk undermining the last decades of research.

There are still a lot of things that we do not know about the mechanisms of mental practice of action. What does each part of the brain engaged in mental practice actually do; how do processes relate to one another; what happens when different areas in the network are damaged? There are indications that insufficient drive to address the fundamentals of mental practice is starting to become a real issue of concern. The systematic review in this issue by Braun et al. (2013) concludes that the clinical evidence for mental practice efficacy in neuro-rehabilitation is weakening. The reasons for this seems to be the lack of theory-driven interventions, conceptual confusion (what does mental practice actually entail in practice?) and general methodological malaise including feasibility, dose, responders/non-responders, and adherence issues in larger scale trials that are more representative of clinical practice. Alternatively, when neuroscience evidence is carefully implemented in theory-driven clinical evaluation of mental practice, this may not translate to earlier reported clinical benefit (Ietswaart et al., 2011). Indeed, Malouin et al. (2013) in this issue highlight significant issues with the translation of experimental findings into clinical practice. Malouin et al.'s critical review is constructive, however, by suggesting ways in which the value of mental practice can be redeemed by addressing underlying mechanisms of mental practice efficacy. They conclude that the field must now truly put the use of mental practice to the test. Mental practice may indeed benefit the large number of stroke patients in neuro-rehabilitation, but unless mental practice is truly put to the test, this application may be superseded by other clinical innovations, for example, robotic assisted therapy. The field needs to deliver the necessary clarity on what exactly are the "active ingredients" of mental practice; what are the things that do not work and are mere distractions; which complexities play a role. Only then can we formulate effective guidance on what mental practice should actually entail in clinical practice. In the meantime, mental practice therapy in neuro-rehabilitation is already currently recommended treatment in many clinical guidelines. This current position means that we need to act fast in order to understand the processes and benefits of mental practice for clinical use. However, the current questionable guidance, range of possible uses, lack of efficacy etc. will likely undermine clinicians' willingness to adopt the treatment in the forthcoming years unless some clarity emerges.

Currently, much of the research effort goes to further documenting the correlates of mental practice, i.e., the fact that imagery and observation resonate with other motoric processes. In that respect, a number of the studies reported in this issue are exceptions to this rule in the way these studies ambitiously delineate the mental practice process by for example comparing the quasi-visual and the verbal-cognitive element of mental practice efficacy (Saimpont et al., 2013, this issue), or by contrasting the efficacy of different visual perspectives in mental practice (Callow et al., 2013, this issue; Yao et al., 2013, this issue), or by separating the impact of active imagery and passive observation (Eaves et al., 2014, this issue). It is an issue of concern, however, that such experimental approaches are generally not pursued (nor funded) as part of clinical evaluations, when now is the time to establish the finer details of mental practice efficacy in clinical contexts. We therefore advocate more high risk, high gain evaluations of mental practice that can establish the real impact of mental practice on the lives of real people in the clinic.

Further to bringing clarity with regards to the underlying mechanisms of mental practice, there is a real need to establish the modes of delivery and dosage. Clinicians furthermore need tools to make predictions of which patients will benefit and from what types of mental practice treatment. Lack of clarity on patient characteristics such as motor imagery ability can easily lead to miss-use of current findings exposing a risk of clinicians dismissing patients who they believe would not stand to benefit from mental practice-based rehabilitation. It would be great if we could say with some level of certainty whether a braindamaged patient has an intact ability to use and benefit from mental practice therapies. Some authors would claim this can be done either through subjective methods such as vividness questionnaires, or through more objective methods such as mental chronometry (Milner, 1986), or monitoring automatic covert action simulation such as the cognitive hand mental rotation task established by Parsons (1987), or the response of the autonomic nervous system in mental practice as proposed by Collet et al. (2013, this issue). There is pressure on the research community to provide reliable measures of motor imagery ability on which clinicians can base a decision whether to provide a patient with mental practice rehabilitation. But quite possibly we do not (yet) have reliable tools on which such important decisions can be based. A study by de Vries et al. (2013, this issue) documenting motor imagery ability in stroke patients, showed that poor motor imagery ability as measured by subjective vividness questionnaires was not associated with poor performance also on objective imagery ability assessment. So although vividness scores suggested the patients had poor motor imagery, objective task performance in these stroke patients suggested that motor imagery was in fact intact. This situation could lead to the risk that clinicians when using only vividness scores could dismiss patients as poor imagers and therefore unable to benefit from mental practice-based rehabilitation, while the patients' imagery ability would be deemed intact if measured in other ways. Although Lawrence et al. (2013, this issue) report that high motor imagery vividness is associated with an increased benefit of mental practice in novice gymnasts compared to the lower performance gains in those with low motor imagery 
vividness, this relationship may not be a simple one suitable for rehabilitation treatment decisions.

This research topic aimed to address confusion regarding the concepts of imagery and observation which has hampered the progression of mental practice research both scientifically and in translation to clinical practice. Wondrusch and SchusterAmft (2013, this issue) remarkably point to the need to address any confusion regarding mental practice even at a therapeutic level. They advocate a good understanding of theory and practice in recipients using mental practice rehabilitation techniques by describing ways to teach stroke patients mental practice. Other contributions in this issue broaden the concept of mental practice in a number of ways, such as Howatson et al.'s rationale for including the observation of one's own movements within the mental practice concept (Howatson et al., 2013, this issue), Smith and Wakefield's considerations with regards to the timing rate of mental practice (Smith and Wakefield, 2013, this issue), Kirsch et al.'s link between action simulation and aesthetic experience (Kirsch et al., 2013, this issue), Schack et al.'s novel theory of how mental practice develops cognitive mental representation structures (Schack et al., 2014, this issue), and importantly Vogt et al.'s meticulous review of the evidence of why mental practice should encompass both motor imagery and action observation (Vogt et al., 2013, this issue).

Because neuroimaging studies provide strong evidence for action simulation, but the link to behavioral change is perhaps weak, we invited contributions to show that mental practice efficacy might be driven by neuroplasticity processes evoked

\section{REFERENCES}

Braun, S., Kleynen, M., van Heel, T., Kruithof, N., Wade, D., and Beurskens, A. (2013). The effects of mental practice in neurological rehabilitation; a systematic review and meta-analysis. Front. Hum. Neurosci. 7:390. doi: 10.3389/fnhum.2013.00390

Callow, N., Roberts, R., Hardy, L., Jiang, D., and Edwards, M. G. (2013). Performance improvements from imagery: evidence that internal visual imagery is superior to external visual imagery for slalom performance. Front. Hum. Neurosci. 7:697. doi: 10.3389/fnhum.2013.00697

Collet, C., Di Rienzo, F., El Hoyek, N., and Guillot, A. (2013). Autonomic nervous system correlates in movement observation and motor imagery. Front. Hum. Neurosci. 7:415. doi: 10.3389/fnhum.2013.00415

de Vries, S., Tepper, M., Feenstra, W., Oosterveld, H., Boonstra, A. M., and Otten, B. (2013). Motor imagery ability in stroke patients: the relationship between implicit and explicit motor imagery measures. Front. Hum. Neurosci. 7:790. doi: 10.3389/fnhum.2013.00790

Eaves, D. L., Haythornthwaite, L., and Vogt, S. (2014). Motor imagery during action observation modulates automatic imitation effects in rhythmical actions. Front. Hum. Neurosci. 8:28. doi: 10.3389/fnhum.2014.00028

Howatson, G., Zult, T., Farthing, J. P., Zijdewind, I., and Hortobagyi, T. (2013). Mirror training to augment cross-education during resistance training: a hypothesis. Front. Hum. Neurosci. 7:396. doi: 10.3389/fnhum.2013. 00396

Ietswaart, M., Johnston, M., Dijkerman, H. C., Joice, S., Scott, C. L., MacWalter, R. S., et al. (2011). Mental practice with motor imagery in stroke recovery: randomized controlled trial of efficacy. Brain 134, 1373-1386. doi: 10.1093/brain/awr077

Jackson, P. L., Lafleur, A. F., Malouin, F., Richards, C., and Doyon, J. (2001). Potential role of mental practice using motor imagery in by action simulation. The preliminary work by Olsson and Lundstrom (2013, this issue) shows that successful action anticipation, as a precursor of mental practice, appeared associated with motor and temporal regions of the brain. Future research needs to investigate evidence of the associations between mental practice performance benefits and brain plasticity in the motor network. It is possible that combination of techniques is needed, including functional magnetic resonance imaging (fMRI), diffusion tensor imaging (DTI), MEG, and EEG.

In conclusion, in an attempt to build on interdisciplinary consensus on the nature and application of mental practice, this research topic integrated perspectives from the full range of the disciplines involved in mental practice research. It furthermore intentionally did not seek to limit mental practice to a narrow interpretation of conscious mental rehearsal of movement or motor imagery, but instead advocates to include imitation and action observation of self or others as an interpretation of mental practice as Action Simulation Therapy (AST). Such an interpretation of AST mental practice is justified in light of the evidence for neural equivalence. What the neuroscience of neural equivalence means for our understanding of behavior, mechanisms, and applied efficacy of mental practice, however, needs a much more sustained research effort devoid of complacency and supported by high-risk-high-gain research funding. With this shared and funded research drive it will be possible to accelerate our understanding and agreement on the core processes of mental practice, and therefore speed up the translation of evidence-based benefit of applied use of mental practice in sport and clinical practice.

neurologic rehabilitation. Arch. Phys. Med. Rehabil. 82, 1133-1141. doi: 10.1053/apmr.2001.24286

Jeannerod, M. (1994). The representing brain - neural correlates of motor intention and imagery. Behav. Brain Sci. 17, 187-202. doi: 10.1017/S0140525X0 0034026

Kirsch, L. P., Drommelschmidt, K. A., and Cross, E. S. (2013). The impact of sensorimotor experience on affective evaluation of dance. Front. Hum. Neurosci. 7:521. doi: 10.3389/fnhum.2013.00521

Lawrence, G., Callow, N., and Roberts, R. (2013). Watch me if you can: imagery ability moderates observational learning effectiveness. Front. Hum. Neurosci. 7:522. doi: $10.3389 /$ fnhum.2013.00522

Malouin, F., Jackson, P. L., and Richards, C. L. (2013). Towards the integration of mental practice in rehabilitation programs. A critical review. Front. Hum. Neurosci. 7:576. doi: 10.3389/fnhum.2013.00576

Milner, A. D. (1986). Chronometric analysis in neuropsychology. Neuropsychologia 24, 115-128.

Olsson, C., and Lundstrom, P. (2013). Using action observation to study superior motor performance: a pilot fMRI study. Front. Hum. Neurosci. 7:819. doi: 10.3389/fnhum.2013.00819

Parsons, L. M. (1987). Imagined spatial transformations of one's hands and feet. Cogn. Psychol. 19, 178-241. doi: 10.1016/0010-0285(87)90011-9

Rizzolatti, G., and Craighero, L. (2004). The mirror-neuron system. Annu. Rev. Neurosci. 27, 169-192. doi: 10.1146/annurev.neuro.27.070203.144230

Saimpont, A., Lafleur, M. F., Malouin, F., Richards, C. L., Doyon, J., and Jackson, P. L. (2013). The comparison between motor imagery and verbal rehearsal on the learning of sequential movements. Front. Hum. Neurosci. 7:773. doi: 10.3389/fnhum.2013.00773

Schack, T., Essig, K., Frank, C., and Koester, D. (2014). Mental representation and motor imagery training. Front. Hum. Neurosci. 8:328. doi: 10.3389/fnhum.2014.00328 
Sharma, N., and Baron, J. (2013). Does motor imagery share neural networks with executed movement: a multivariate fMRI analysis. Front. Hum. Neurosci. 7:564. doi: 10.3389/fnhum.2013.00564

Smith, D., and Wakefield, C. (2013). A timely review of a key aspect of motor imagery: a commentary on Guillot et al.(2012). Front. Hum. Neurosci. 7:761. doi: 10.3389/fnhum.2013.00761

Vogt, S., Di Rienzo, F., Collet, C., Collins, A., and Guillot, A. (2013). Multiple roles of motor imagery during action observation. Front. Hum. Neurosci. 7:807. doi: 10.3389/fnhum.2013.00807

Wondrusch, C., and Schuster-Amft, C. (2013). A standardized motor imagery introduction program (MIIP) for neuro-rehabilitation: development and evaluation. Front. Hum. Neurosci. 7:477. doi: 10.3389/fnhum.2013.00477

Yao, W. X., Ranganathan, V. K., Allexandre, D., Siemionow, V., and Yue, G. H. (2013). Kinesthetic imagery training of forceful muscle contractions increases brain signal and muscle strength. Front. Hum. Neurosci. 7:561. doi: 10.3389/fnhum.2013. 00561

Conflict of Interest Statement: The authors declare that the research was conducted in the absence of any commercial or financial relationships that could be construed as a potential conflict of interest.

Copyright (c) 2015 Ietswaart, Butler, Jackson and Edwards. This is an open-access article distributed under the terms of the Creative Commons Attribution License (CC $B Y)$. The use, distribution or reproduction in other forums is permitted, provided the original author(s) or licensor are credited and that the original publication in this journal is cited, in accordance with accepted academic practice. No use, distribution or reproduction is permitted which does not comply with these terms. 